\title{
Author Index Volume 10 (2016)
}

Akyuz, Y. C., see Ergun, H.

3 (2016) 379

Alashri, S., Alzahrani, S., Tsai, J.-Y., Corman, S. R. \& Davulcu, H., "Climate change" frames detection and categorization based on generalized concepts

2 (2016) 147

Alexiou, G., see Papadakis, N.

3 (2016) 399

Allahyari, M. \& Kochut, K., Using semantically-extended LDA topic model for semantic tagging

4 (2016) 503

Allen, C. M., see Cao, H.

3 (2016) 299

Alzahrani, S., see Alashri, S.

2 (2016) 147

Armiato, D. A., Yano, Y., de Faveri, V. Z. \& Guido, R. C., Handwritten signatures verification through their acoustic patterns based on the discrete wavelet-packet transform and semantic-matching classifiers

Atsalaki, X., see Papadakis, N.

4 (2016) 557

3 (2016) 399

Auer, S., see Halilaj, L.

2 (2016) 167

Bagheri, E., see Ghashghaei, M.

1 (2016) 121

Ba-Hutair, M. N., see Kamel, I.

3 (2016) 347

Beecks, C., Hassani, M., Brenger, B., Hinnell, J., Schüller, D., Mittelberg, I. $\&$ Seidl, T., Efficient query processing in $3 \mathrm{D}$ motion capture gesture databases

$1(2016) 5$

Brenger, B., see Beecks, C.

$1(2016) 5$

Cao, H., Li, Y., Allen, C. M., Phinney, M. A. \& Shyu, C.-R., Visual reasoning indexing and retrieval using in-memory computing

3 (2016) 299

Chen, Y., Wang, W., He, W. \& Li, X., An empirical study of the textual content of online videos

3 (2016) 323

Choi, K.-S., see Poon, G.

3 (2016) 365

Corman, S. R., see Alashri, S.

2 (2016) 147

Coskun, G., see Halilaj, L.

2 (2016) 167

Cuzzola, J., see Ghashghaei, M.

1 (2016) 121

Davulcu, H., see Alashri, S.

2 (2016) 147

de Faveri, V. Z., see Armiato, D. A.

4 (2016) 557

Deligiannis, I. A., see Papadakis, N.

3 (2016) 399 
Eckert, K., see Hartmann, T.

$2(2016) 193$

Ergun, H., Akyuz, Y. C., Sert, M. \& Liu, J., Early and late level fusion of deep convolutional neural networks for visual concept recognition

3 (2016) 379

Freitas, A., see Mehdi, M.

2 (2016) 219

Ghashghaei, M., Bagheri, E., Cuzzola, J., Ghorbani, A. A. \& Noorian, Z., Semantic disambiguation and linking of quantitative mentions in textual content

$1(2016) 121$

Ghorbani, A. A., see Ghashghaei, M.

1 (2016) 121

Goto, M., see Nakano, T.

1 (2016) 27

Grangel-González, I., see Halilaj, L.

2 (2016) 167

Guido, R. C., see Armiato, D. A.

4 (2016) 557

Halilaj, L., Grangel-González, I., Coskun, G., Lohmann, S. \& Auer, S., Git4Voc: Collaborative vocabulary development based on Git

Hao, X., Zhang, G. \& Ma, S., Deep learning

3 (2016) 417

Hartmann, T., Zapilko, B., Wackerow, J. \& Eckert, K., Directing the development of constraint languages by checking constraints on RDF data

2 (2016) 193

Hassani, M., see Beecks, C.

1 (2016) 5

He, W., see Chen, Y.

3 (2016) 323

Hinnell, J., see Beecks, C.

$1(2016) 5$

Huber, S., Seiger, R., Kühnert, A., Theodorou, V. \& Schlegel, T., Goalbased semantic queries for dynamic processes in the Internet of Things

2 (2016) 269

Jacques Jr., J. C. S. \& Musse, S. R., Shape-based pedestrian segmentation in still images

$1(2016) 53$

Jares, J., see Mehdi, M.

2 (2016) 219

Jha, A. K., see Mehdi, M.

2 (2016) 219

Kalaentzis, A., see Papadakis, N.

3 (2016) 399

Kamel, I. \& Ba-Hutair, M. N., Privacy protection in outsourced spatial databases

3 (2016) 347

Kawamura, T., see Yokoo, R.

4 (2016) 445

Khan, Y., see Mehdi, M.

2 (2016) 219

Kühnert, A., see Huber, S.

2 (2016) 269

Kim, M., Wang, S.-T., Ostrowski, D., Rychtyckyj, N. \& Macneille, P., Federated ontologies and industrial applications

1 (2016) 101

Kochut, K., see Allahyari, M.

Komporakis, I., see Papadakis, N.

4 (2016) 503

3 (2016) 399

Kondylakis, H., see Papadakis, N.

3 (2016) 399

Kwan, K. C., see Poon, G.

3 (2016) 365

Li, Q., Li, T., Xia, B., Ni, M., Liu, X., Zhou, Q. \& Qi, Y., First: Face Identity Recognition in $\underline{\text { SmarT }}$ bank 
Li, T., see Li, Q.

4 (2016) 569

Li, X., see Chen, Y.

3 (2016) 323

Li, Y., see Cao, H.

3 (2016) 299

Liu, J., see Ergun, H.

3 (2016) 379

Liu, X., see Li, Q.

4 (2016) 569

Lohmann, S., see Halilaj, L.

2 (2016) 167

Macneille, P., see Kim, M.

1 (2016) 101

Ma, S., see Hao, X.

Mehdi, M., Khan, Y., Jares, J., Freitas, A., Jha, A. K., Sakellarios, A. \&

3 (2016) 417

Sahay, R., A linked data visualiser for finite element biosimulations

2 (2016) 219

Mittelberg, I., see Beecks, C.

$1(2016) 5$

Musse, S. R., see Jacques Jr., J. C. S.

$1(2016) 53$

Nakano, T., Yoshii, K. \& Goto, M., Musical similarity and commonness estimation based on probabilistic generative models of musical elements

1 (2016) 27

$\mathrm{Ni}, \mathrm{M}$., see Li, Q.

Noorian, Z., see Ghashghaei, M.

4 (2016) 569

1 (2016) 121

Ohsuga, A., see Yokoo, R.

4 (2016) 445

Ostrowski, D., see Kim, M.

1 (2016) 101

Panangadan, A. V., see Patri, O. P.

$4(2016) 461$

Pang, W.-M., see Poon, G.

Papadakis, N., Kondylakis, H., Kalaentzis, A., Komporakis, I.,

3 (2016) 365

Deligiannis, I. A., Steiakaki, M., Alexiou, G. \& Atsalaki, X., BlogSearch:

Semantic services for aggregating and searching blog articles

3 (2016) 399

Patri, O. P., Panangadan, A. V., Sorathia, V. S. \& Prasanna, V. K., Sensors to events: Semantic modeling and recognition of events from data streams

4 (2016) 461

3 (2016) 299

Poon, G., Kwan, K. C., Pang, W.-M. \& Choi, K.-S., Towards using tiny
Peyney, M. A., see Cao, H. sensors with heat balancing criteria for child care reminders

3 (2016) 365

Prasanna, V. K., see Patri, O. P.

4 (2016) 461

Qi, Y., see Li, Q.

4 (2016) 569

Rychtyckyj, N., see Kim, M.

$1(2016) 101$

Sahay, R., see Mehdi, M.

2 (2016) 219

Sakellarios, A., see Mehdi, M.

2 (2016) 219

Schlegel, T., see Huber, S.

2 (2016) 269

Schüller, D., see Beecks, C.

1 (2016) 5

Seidl, T., see Beecks, C.

1 (2016) 5 
Seiger, R., see Huber, S.

2 (2016) 269

Sert, M., see Ergun, H.

3 (2016) 379

Shyu, C.-R., see Cao, H.

3 (2016) 299

Shyu, M.-L., see Yan, Y.

2 (2016) 247

Singh, K., User reachability in islands of WeBRTC communication apps

1 (2016) 73

Sorathia, V. S., see Patri, O. P.

4 (2016) 461

Stanchev, L., Fine-tuning an algorithm for semantic document clustering using a similarity graph

4 (2016) 527

Steiakaki, M., see Papadakis, N.

3 (2016) 399

Theodorou, V., see Huber, S.

2 (2016) 269

Tsai, J.-Y., see Alashri, S.

2 (2016) 147

Wackerow, J., see Hartmann, T.

$2(2016) 193$

Wang, S.-T., see Kim, M.

1 (2016) 101

Wang, W., see Chen, Y.

3 (2016) 323

Xia, B., see Li, Q.

$4(2016) 569$

Yan, Y., Shyu, M.-L. \& Zhu, Q., Supporting semantic concept retrieval with negative correlations in a multimedia big data mining system

2 (2016) 247

Yano, Y., see Armiato, D. A.

4 (2016) 557

Yokoo, R., Kawamura, T. \& Ohsuga, A., Semantics-based news delivering service

$4(2016) 445$

Yoshii, K., see Nakano, T.

1 (2016) 27

Zapilko, B., see Hartmann, T.

2 (2016) 193

Zhang, G., see Hao, X.

3 (2016) 417

Zhou, Q., see Li, Q.

4 (2016) 569

Zhu, Q., see Yan, Y.

2 (2016) 247 